\title{
SOME APPROACHES TO ERGODIC AND ADAPTIVE CONTROL OF STOCHASTIC SEMILINEAR SYSTEMS
}

\author{
T. E. Duncan \\ Department of Mathematics, University of Kansas \\ Lawrence, KS 66045 \\ U.S.A.* \\ duncan@math.ukans.edu
}

\begin{abstract}
Some ergodic and adaptive control problems for stochastic semilinear systems are described. The stochastic systems have cylindrical noise processes and the control is either distributed or boundary/point. The development of adaptive control for stochastic distributed parameter systems is described commencing with adaptive control problems for linear stochastic systems with quadratic cost functionals.
\end{abstract}

\section{INTRODUCTION}

The study of adaptive control of stochastic distributed systems has developed primarily in this decade. For adaptive control, the systems are only partially known and they must be controlled so there are the problems of identification and control. For the control part it is clear that some results for the control of the completely known systems should be available, so it is natural that there is some lag time between the results for optimal control and adaptive control of these systems. If it is desired that an adaptive control achieves the optimal cost for the control of the known system then an ergodic cost criterion is natural. If an adaptive control achieves this optimal cost then it is called self optimal. The semigroup approach has been an important method for the analysis of distributed parameter systems. The initial work on adaptive control using the semigroup approach is done in [2] where an adaptive control problem of a partially known linear stochastic system, for example, a stochastic partial differential equation, with a distributed control and a quadratic cost functional is solved. Since it seems to be more natural to use boundary or point control,

*Research supported in part by NSF Grant DMS 9623439.

The original version of this chapter was revised: The copyright line was incorrect. This has been corrected. The Erratum to this chapter is available at DOI: 10.1007/978-0-387-35359-3_40 
instead of distributed control, some adaptive control problems for partially known linear stochastic distributed parameter systems with boundary/point control and quadratic cost functionals are solved in [3, 4]. These adaptive control problems use the optimal controls for deterministic, linear distributed parameter systems with infinite time horizon, quadratic cost functionals. It is well known, at least for finite dimensional systems, that the optimal control for the deterministic system gives the optimal control of the stochastic system with the corresponding ergodic cost. However this relation assumes that the systems are linear and the cost functional is quadratic.

For stochastic semilinear systems the situation is significantly more complicated. There are questions of existence and uniqueness of the solutions of the stochastic differential equations that describe the stochastic semilinear systems. The transition measures and semigroup for the solution of a stochastic semilinear system with a Markov-type control have to be shown to be strongly Feller, tight and irreducible so that invariant measures exist and are unique. These results are important for the ergodic control problem. For the adaptive control problem it is important to verify the continuity of the optimal cost and the optimal control with respect to parameters. These properties are important because typically a strongly consistent family of estimates of the unknown parameters is constructed and it is desired to show that a certainty equivalence adaptive control, that is obtained by using the estimate of the unknown parameter in place of the true parameter in the optimal control, converges in some sense to the optimal control and the "running" costs for this adaptive control converge to the optimal ergodic cost for the known system.

For modelling of the controlled stochastic semilinear system there are distributed and boundary/point control. For boundary/point control some results have been obtained for ergodic control and continuity properties for adaptive control in [6]. While these results provide important information for both ergodic and adaptive control, an optimal control is not given explicitly so that the continuity of an optimal control with respect to parameters is not known. Thus, if a certainty equivalence adaptive control is used with a strongly consistent family of estimates of the unknown parameter, then it is not clear that these adaptive controls converge to an optimal control for the true system. For distributed control of parameter dependent semilinear stochastic systems, some results have been obtained for ergodic control and continuity properties for adaptive control in [5]. However, an optimal control is not given explicitly, so it is difficult to verify that a family of adaptive controls converges to an optimal control. Another approach to ergodic cost, distributed control is given in [8]. While these results have potential application to adaptive control, it seems that more information is required about an optimal control.

For some ergodic, distributed control problems of stochastic semilinear systems, a Hamilton-Jacobi-Bellman equation is solved and an optimal control is given in [9]. In [7] the results in [9, 10] are used as a starting point for the verification of continuity properties of the optimal cost and an optimal control with respect to parameters. These results are used to solve an adaptive 
control problem. Some of these results are described explicitly in this paper. The proofs of the results that are given here are contained in [7]. The noise process that occurs in these semilinear stochastic systems is cylindrical, that is, it does not exist in the Hilbert space where the state of the system is described. Thus the semigroup associated with the linear part of the semilinear system is required to regularize this cylindrical noise.

\section{PRELIMINARIES}

A partially known stochastic semilinear system is described with a distributed control.

Let $(X(t), t \geq 0)$ be an $H$-valued, parameter dependent, controlled process that satisfies the (formal) stochastic differential equation

$$
\begin{aligned}
d X(t) & =(A X(t)+f(\alpha, X(t))-u(t)) d t+Q^{1 / 2} d W(t) \\
X(0) & =x
\end{aligned}
$$

where $H$ is a real, separable Hilbert space with inner product $\langle\cdot, \cdot\rangle$ and norm $|\cdot|, A: \operatorname{Dom}(A) \rightarrow H$ is a densely defined, unbounded linear operator on $H, f(\alpha, \cdot): H \rightarrow H$ for each $\alpha \in \mathcal{A} \subset \mathbb{R}^{d}$ that is a compact set of parameters, $(W(t), t \geq 0)$ is a standard, cylindrical $H$-valued Wiener process defined on a filtered probability space $\left(\Omega, \mathcal{F},\left(\mathcal{F}_{t}\right), \mathbb{P}\right)$ and $Q \in \mathcal{L}(H)$. The family of admissible controls is

$$
\mathcal{U}=\left\{u: \mathbb{R}_{+} \times \Omega \rightarrow B_{R} \mid u \text { is measurable and }\left(\mathcal{F}_{t}\right) \text { adapted }\right\}
$$

where $B_{R}=\{y \in H|| y \mid \leq R\}$ and $R>0$ is fixed. A family of Markov controls, e.g., $u(t)=\tilde{u}(X(t))$, is also considered where $\tilde{u} \in \tilde{\mathcal{U}}$ and

$$
\tilde{\mathcal{U}}=\left\{\tilde{u}: H \rightarrow B_{R} \mid \tilde{u} \text { is Borel measurable }\right\} .
$$

The cost functionals $J(x, \lambda, u)$ and $\tilde{J}(x, u)$ are given as

$$
J(x, \lambda, u)=\mathbb{E}_{x, u} \int_{0}^{\infty} e^{-\lambda t}(\psi(X(t))+h(u(t))) d t
$$

and

$$
\tilde{J}(x, u)=\liminf _{T \rightarrow \infty} \mathbb{E}_{x, u} \frac{1}{T} \int_{0}^{T}(\psi(X(t))+h(u(t))) d t
$$

where $\lambda>0$ and $h: B_{R} \rightarrow \mathbb{R}_{+}$and $\psi: H \rightarrow \mathbb{R}$ that describe a discounted and an ergodic control problem, respectively.

The following assumptions are selectively used to solve an adaptive control problem.

(A1) The linear operator $Q=Q^{1 / 2^{*}} Q^{1 / 2}$ is invertible, $Q^{-1} \in \mathcal{L}(H)$ and $(S(t), t \geq 0)$, where $S(t)=e^{t A}$, is an exponentially stable semigroup of contractions, that is, $\|S(t)\|_{\mathcal{L}(H)} \leq e^{-\omega t}$ for all $t \geq 0$ and some $\omega>0$. Furthermore, the semigroup is Hilbert-Schmidt and there is a $\gamma>0$ such 
that $\int_{0}^{T} t^{-\gamma}\|S(t)\|_{\mathrm{HS}}^{2} d t<\infty$ for some $T>0$ where $\|\cdot\|_{\mathrm{HS}}$ is the HilbertSchmidt norm.

(A2) The function $f(\alpha, \cdot): H \rightarrow H$ is Lipschitz continuous and Gateaux differentiable. The Gateaux derivative $D f(\alpha, \cdot) h$ is continuous on $H$ for each $h \in H$ and $\alpha \in \mathcal{A}$ and there is a $\beta \in \mathbb{R}$ such that $\langle D f(\alpha, x) h, h\rangle \leq$ $\beta|h|^{2}$ for all $x \in H, h \in H$ and $\alpha \in \mathcal{A}$.

(A3) There are constants $p>0, \theta>0$ and $C>0$ such that $\mid f\left(\alpha_{1}, x\right)-$ $f\left(\alpha_{2}, x\right)|\leq C| \alpha_{1}-\left.\alpha_{2}\right|^{\theta}\left(1+|x|^{p}\right)$ for all $\alpha_{1}, \alpha_{2} \in \mathcal{A}$ and $x \in H$.

(A4) $\psi \in C_{b}(H)$.

(A5) The function $\psi: H \rightarrow \mathbb{R}$ is convex and bounded on bounded sets and continuous. The function $\tilde{H}: H \rightarrow \mathbb{R}$ given by $\tilde{H}(x)=\sup _{|y| \leq R}[\langle y, x\rangle-$ $h(y)]$ is Lipschitz continuous and Gateaux differentiable. Each directional derivative of $\tilde{H}$ is continuous on $H$.

Some implications of the assumptions (A1)-(A5) are described now. For the linear stochastic differential equation associated with (2.1), that is, $f \equiv u \equiv 0$, there are a unique mild solution and a unique invariant Gaussian probability measure, $\mu=N\left(0, Q_{\infty}\right)$ where $Q_{\infty}$ is a trace class operator on $H$. If (A2) is satisfied then (2.1) has a unique mild solution for each $u \in \mathcal{U}$ and $\alpha \in \mathcal{A}$. If the control in (2.1) has the feedback form $u(t)=\tilde{u}(X(t))$ where $\tilde{u} \in \tilde{\mathcal{U}}$ then the solution of (2.1) is obtained by an absolute continuity of measures as a weak solution in the probabilistic sense.

The assumption (A3) is used to verify a suitable continuous dependence of the solutions of the ergodic Hamilton-Jacobi-Bellman equations on the parameter which is important to verify the self-optimality of a certainty equivalence adaptive control. The assumptions (A4) and (A5) are standard conditions on a cost functional in the stochastic control of semilinear systems (e.g., $[9,10])$. Note that (A5) is satisfied in the case where $h(x)=|x|^{2}$ so that $\tilde{H}(x)=\hat{H}(\|x\|)$ where

$$
\hat{H}(r)= \begin{cases}\frac{r^{2}}{4} & \text { if }|r| \leq 2 R \\ R|r|-R^{2} & \text { if }|r|>2 R\end{cases}
$$

To provide some additional perspective for the adaptive control problem, an example of a stochastic partial differential equation that satisfies the assumptions is given. Consider the stochastic PDE

$$
\frac{\partial y}{\partial t}(t, \xi)=\gamma \frac{\partial^{2} y}{\partial \xi^{2}}(t, \xi)+F(\alpha, y(t, \xi))-u(t, \xi)+\eta(t, \xi)
$$

for $(t, \xi) \in \mathbb{R}_{+} \times(0,1)$ with the initial condition $y(0, \xi)=y_{0}(\xi) . \xi \in(0,1)$ and the Dirichlet boundary conditions

$$
y(t, 0)=y(t, 1)=0
$$


for $t \geq 0$ and $\gamma>0$ is a constant, $F: \mathcal{A} \times \mathbb{R} \rightarrow \mathbb{R}$ satisfies $F(\alpha, \cdot) \in C^{1}(\mathbb{R})$ for each $\alpha \in \mathcal{A}, F^{\prime}(\alpha, y) \leq \beta$ and $\left|F\left(\alpha_{1}, y\right)-F\left(\alpha_{2}, y\right)\right| \leq C\left|\alpha_{1}-\alpha_{2}\right|^{\theta}(1+|y|)$ for some constants $\beta \in \mathbb{R}_{+}, C>0$ and all $\alpha, \alpha_{1}, \alpha_{2} \in \mathcal{A}, y \in \mathbb{R}$ and $\eta$ formally denotes a space time white noise. The control $(u(t), t \geq 0)$ is assumed to be adapted to the noise process and to take values in a ball, $B_{R}$, in $L^{2}(0,1)$. The formal equation (2.6) can be rigorously described in a standard way as an equation of the form (2.1) in the Hilbert space $H=L^{2}(0,1), A=\gamma\left(\partial^{2} / \partial \xi^{2}\right)$, $\operatorname{Dom}(A)=\left\{\varphi \in L^{2}(0,1) \mid \varphi, \varphi^{\prime}\right.$ are absolutely continuous, $\varphi^{\prime \prime} \in L^{2}(0,1)$, $\varphi(0)=\varphi(1)=0\}, f(\alpha, x)(\xi)=F(\alpha, x(\xi))$ for $x \in H, \alpha \in \mathcal{A}, \xi \in(0,1)$ and a cylindrical Wiener process with $Q=\delta I$ where $\delta>0$ is a constant and $I$ is the identity on $I$. For $\psi$ and $h$ in the cost functionals $(2.4,2.5)$ arbitrary $\psi \in C_{b}\left(L^{2}(0,1)\right)$ and $h: L^{2}(0,1) \rightarrow \mathbb{R}_{+}$satisfying (A5) can be chosen, e.g., $h(u)=|u|^{2}$. It is well known that all of the assumptions (A1)-(A5) are satisfied where $\gamma \in(0,1 / 4)$ in $(\mathrm{A} 1)$.

\section{MAIN RESULTS}

The formal Hamilton-Jacobi-Bellman equations corresponding to the control problems $(2.1,2.4)$ and $(2.1,2.5)$ are respectively

$$
\begin{aligned}
& \frac{1}{2} \operatorname{Tr} Q D^{2} v_{\alpha}^{\lambda}(x)+\left\langle A x, D v_{\alpha}^{\lambda}(x)\right\rangle+\left\langle f(\alpha, x), D v_{\alpha}^{\lambda}(x)\right\rangle \\
& \quad-\tilde{H}\left(D v_{\alpha}^{\lambda}(x)\right)+\psi(x)=\lambda v_{\alpha}^{\lambda}(x) \\
& \frac{1}{2} \operatorname{Tr} Q D^{2} v_{\alpha}(x)+\left\langle A x, D v_{\alpha}(x)\right\rangle+\left\langle f(\alpha, x), D v_{\alpha}(x)\right\rangle \\
& \quad-\tilde{H}\left(D v_{\alpha}(x)\right)+\psi(x)=\rho(\alpha) .
\end{aligned}
$$

In (3.2) it is necessary to solve for the pair $\left(v_{\alpha}, \rho(\alpha)\right), \rho(\alpha) \in \mathbb{R}$ for each $\alpha \in \mathcal{A}$.

It is clear that the existence of strong solutions to (3.1) and (3.2) cannot be expected because of the first two terms on the left hand side of these equations, that is, $Q$ is not trace class and $A$ is only densely defined in $H$. The approach in [9] is to replace the first two terms in (3.1) and (3.2) by the generator of an Ornstein-Uhlenbeck semigroup in a suitable function space. The results of $[9$, 10] are used but for simplicity the solutions of (3.1) and (3.2) are defined in a weaker sense which is suitable for the applications to adaptive control.

Let $\mu=N\left(0, Q_{\infty}\right)$ be the invariant measure and $\left(R_{t} \varphi\right)(x)=\mathbb{E}_{x} \varphi(Z(t))$ be the Markov transition semigroup for the Ornstein-Uhlenbeck process $(Z(t), t \geq$ $0)$ that is the solution of (2.1) with $f \equiv u \equiv 0$. It is well known that $\left(R_{t}, t \geq 0\right)$ is a strongly continuous semigroup on the Hilbert space $\mathcal{H}=L^{2}(H, \mu)$. Let $\mathcal{L}$ be the infinitesimal generator of the semigroup $\left(R_{t}, t \geq 0\right)$ in $\mathcal{H}$. Furthermore, let $\mathcal{L}_{0}$ be given by

$$
\mathcal{L}_{0} \varphi(x)=\frac{1}{2} \operatorname{Tr} Q D^{2} \varphi(x)+\left\langle x, A^{*} D \varphi(x)\right\rangle
$$

for $x \in H$ and $\varphi \in \operatorname{Dom}\left(\mathcal{L}_{0}\right)$ where $\operatorname{Dom}\left(\mathcal{L}_{0}\right)=\left\{\varphi \in C_{b}^{2}(H) \mid(1 / 2) \operatorname{Tr} Q D^{2} \varphi(\cdot) \in\right.$ $\left.C_{b}(H),\left\langle\cdot, A^{*} D \varphi(\cdot)\right\rangle \in C_{b}(H)\right\}$. 
Let $\varphi \in \operatorname{Dom}\left(\mathcal{L}_{0}\right)$ and use the Itô formula to obtain

$$
\begin{aligned}
(\mathcal{L} \varphi)(x) & =\lim _{t \downarrow 0} \frac{1}{t}\left(R_{t} \varphi(x)-\varphi(x)\right) \\
& =\lim _{t \downarrow 0} \frac{1}{t}\left(\mathbb{E}_{x} \varphi(Z(t))-\varphi(x)\right) \\
& =\left(\mathcal{L}_{0} \varphi\right)(x)
\end{aligned}
$$

so $\mathcal{L}$ is a closed extension of the operator $\mathcal{L}_{0}$. This equality, (3.4), motivates the following definition of solution of (3.1) and (3.2).

Definition 3.1 A function $v_{\alpha}^{\lambda} \in \operatorname{Dom}(\mathcal{L})$ and a pair $\left(v_{\alpha}, \rho(\alpha)\right) \in \operatorname{Dom}(\mathcal{L}) \times \mathbb{R}$ are solutions to (3.1) and (3.2) respectively if

$$
\mathcal{L} v_{\alpha}^{\lambda}+\left\langle f(\alpha, \cdot), D v_{\alpha}^{\lambda}\right\rangle-\tilde{H}\left(D v_{\alpha}^{\lambda}\right)+\psi=\lambda v_{\alpha}^{\lambda}
$$

and

$$
\mathcal{L} v_{\alpha}+\left\langle f(\alpha, \cdot), D v_{\alpha}\right\rangle-\tilde{H}\left(D v_{\alpha}\right)+\psi=\rho(\alpha)
$$

are satisfied.

This definition of the solutions to (3.1) and (3.2) requires only that the solutions be in $\operatorname{Dom}(\mathcal{L}) \subset L^{2}(H, \mu)$ so the equations (3.5) and (3.6) are understood in an $L^{2}(H, \mu)$ sense. This relatively weak notion of solution is used to avoid some technical complications. Some results on the solutions to (3.1) and (3.2) are given in [9] and [10]. It is shown that the solutions are more regular than that required in the Definition 1.3.1. For the following two propositions the parameter $\alpha \in \mathcal{A}$ is fixed.

Proposition 3.1 If (A1), (A2), (A4) and (A5) are satisfied, then the equation (3.1) has one and only solution $v_{\alpha}^{\lambda}$ in $\operatorname{Dom}(\mathcal{L}) \cap C_{b}^{1}(H)$. Furthermore,

$$
v_{\alpha}^{\lambda}(x)=\inf _{u \in \mathcal{U}} J(x, \lambda, u)
$$

so that $v_{\alpha}^{\lambda}$ gives the optimal cost and an optimal control in feedback form is $\hat{u}_{\alpha}^{\lambda}(x)=\tilde{D} \tilde{H}\left(D v_{\alpha}^{\lambda}(x)\right)$ for the discounted cost control problem $(2.1,2.4)$.

This proposition has been basically proven by Gozzi and Rouy [10] when $f(\alpha, \cdot)$ is bounded. The generalization in Proposition 1.3.1 has been done by Goldys and Maslowski [9].

The ergodic control problem is usually considered to be more difficult than the discounted control problem because the Hamilton-Jacobi-Bellman equation (3.2) has an intrinsic degeneracy, that is, there is no uniqueness of the solution to (3.2) because if $\left(v_{\alpha}, \rho(\alpha)\right)$ is a solution of $(3.2)$ then $\left(v_{\alpha}+c, \rho(\alpha)\right)$ for $c \in \mathbb{R}$ is also a solution.

Let $R>0$ in (A5), satisfy

$$
R<\frac{\sqrt{\omega_{1}}}{\left|Q^{-1 / 2}\right|_{\mathcal{L}(H)} k\left(\omega_{1}\right) \sqrt{\pi}}
$$


where $\omega-\beta>0, \omega_{1} \in(0, \omega-\beta)$ is fixed and $k\left(\omega_{1}\right)>0$ is a constant depending only on $\omega_{1}$ that is obtained from an upper bound on the Fréchet derivative of the Markov transition semigroup induced by the solution of (2.1) with $u \equiv 0$.

The following proposition is a " $W^{1,2}(H, \mu)$-version" of a result in [9] on the existence and the uniqueness of a solution to the ergodic Hamilton-JacobiBellman equation. While the existence result is weaker than the one in [9], the family of solutions for uniqueness is enlarged. The parameter $\alpha \in \mathcal{A}$ in the following proposition is fixed and

$$
\begin{aligned}
\mathcal{W}=\left\{\varphi \in W^{1,2}(H, \mu)\right. & \mid \varphi \in \operatorname{Dom}(\mathcal{L}),\|D \varphi\|<\infty \\
& |\varphi(x)|+|\mathcal{L} \varphi(x)| \leq k\left(1+|x|^{q}\right) \text { for all } x \in H \\
& \text { and some positive real numbers } k \text { and } q\} .
\end{aligned}
$$

Proposition 3.2 If (A1), (A2), (A4), (A5) with $\omega-\beta>0$ and (3.8) are satisfied then there is a unique solution $\left(v_{\alpha}, \rho(\alpha)\right) \in(\mathcal{W} \times C(H)) \times \mathbb{R}$ of $(3.2)$ such that $v_{\alpha}(0)=0$. Furthermore,

$$
\rho(\alpha)=\inf _{u \in \mathcal{U}} \tilde{J}(x, u)
$$

so that $\rho(\alpha)$ is the optimal cost and an optimal control in feedback form is $\hat{u}_{\alpha}(x)=D H\left(D v_{\alpha}(x)\right)$ for the ergodic control problem $(2.1,2.5)$.

Consider the equation (2.1) with the true parameter value $\alpha_{0} \in \mathcal{A}$, that is,

$$
\begin{aligned}
d X(t) & =\left(A X(t)+f\left(\alpha_{0}, X(t)\right)-\tilde{u}(t)\right) d t+Q^{1 / 2} d W(t) \\
X(0) & =x
\end{aligned}
$$

where

$$
\tilde{u}(t)=D \tilde{H}\left(D v_{\alpha(t)}(X(t))\right)
$$

where $(\alpha(t), t \geq 0)$ is an adapted, measurable, $\mathcal{A}$-valued process satisfying

$$
\lim _{t \rightarrow \infty} \alpha(t)=\alpha_{0} \quad \text { a.s. } \mathbb{P}
$$

and $v_{\alpha}$ is given in Proposition 1.3.2. For notational simplicity, let $v=v_{\alpha_{0}}$ and $\rho=\rho\left(\alpha_{0}\right)$.

Proposition 3.3 If (A1)-(A5) are satisfied, $\omega-\beta>0$ and the inequality (3.8) are satisfied, then

$$
\tilde{J}(x, \tilde{u})=\rho\left(\alpha_{0}\right)
$$

for each $x \in H$, that is, the adaptive control $\tilde{u}$ given by (3.10) is self-optimizing for the ergodic control problem $(2.1,2.5)$. 


\section{References}

[1] G. Da Prato and J. Zabczyk, Stochastic Equations in Infinite Dimensions, Cambridge Univ. Press, Cambridge, 1992.

[2] T. E. Duncan, B. Goldys and B. Pasik-Duncan, Adaptive control of linear stochastic evolution systems, Stochastics Stochastic Rep. 36(1991), 71-90.

[3] T. E. Duncan, B. Maslowski, and B. Pasik-Duncan, Adaptive boundary and point control of linear stochastic distributed parameter systems, SIAM J. Control Optim. 32(1994), 648-672.

[4] T. E. Duncan, B. Maslowski, and B. Pasik-Duncan, Adaptive boundary control of linear distributed parameter systems described by analytic semigroups, Appl. Math. Optim. 33(1996), 107-138.

[5] T. E. Duncan, B. Maslowski, and B. Pasik-Duncan, Ergodic distributed control for parameter dependent stochastic semilinear systems in Hilbert space, Proc. Conf. Stochastic Differential and Differentia Equations, Birkhäuser, 1997.

[6] T. E. Duncan, B. Maslowski, and B. Pasik-Duncan, Ergodic boundary/point control of stochastic semilinear systems, SIAM J. Control Optim. 36(1998), 1020-1047.

[7] T. E. Duncan, B. Maslowski, and B. Pasik-Duncan, Adaptive control for semilinear stochastic systems, preprint.

[8] T. E. Duncan, B. Pasik-Duncan and L. Stettner, On ergodic control of stochastic evolution equations, Stochastic Process. Appl. 15(1997), 723750 .

[9] B. Goldys and B. Maslowski, Ergodic control of semilinear stochastic equations and Hamilton-Jacobi equations, Statistics Dept., Univ. New South Wales, Rept. S97-13.

[10] F. Gozzi and E. Rouy, Regular solutions to second-order stationary Hamilton-Jacobi equations, J. Differential Equations 130(1996), 201-234. 